\title{
MODELAGEM DINÂMICA ESPACIAL COMO FERRAMENTA PARA SIMULAÇÃO DE CENÁRIOS DA PAISAGEM NA REGIÃO PORTUÁRIA DA BAIXADA SANTISTA
}

\author{
Spatial dynamic modeling as a tool for simulating landscape scenarios in the \\ port region of Baixada Santista
}

\author{
Renata Sayuri Kawashima ${ }^{1}$ \\ Mariana Abrantes Giannotti ${ }^{1}$ \\ Cláudia Maria de Almeida ${ }^{2}$ \\ José Alberto Quintanilha ${ }^{1}$
}

\begin{abstract}
Universidade de São Paulo - USP, Escola Politécnica, Departamento de Engenharia de Transportes, Programa de Pós Graduação em Engenharia de Transportes - PPGET. Laboratório de Geoprocessamento. Av. Profo Almeida Prado, 83. Caixa Postal 61548 - CEP 05508-070 - São Paulo, SP, Brasil 1 .Email: resao_sayuri@hotmail.com; mariana.giannotti@usp.br; jaquinta@usp.br Instituto Nacional de Pesquisas Espaciais - INPE. Av. dos Astronautas, 1.758. Caixa Postal 515- CEP 12227-010 - São José dos Campos, SP, Brasil 2

Email: almeida@dsr.inpe.br
\end{abstract}

\section{Resumo:}

Considerando as pressões do setor de infraestrutura portuária sobre as configurações na forma de uso e planejamento do espaço na região portuária da Baixada Santista, tais como o aporte por áreas de retroporto, o armazenamento e o transporte de cargas, este estudo buscou desenvolver modelos dinâmicos da paisagem e investigar a relação entre as alterações no uso e cobertura da terra e a infraestrutura de transportes na região portuária da Baixada Santista-SP. A partir de produtos de sensoriamento remoto e da metodologia de modelagem dinâmica espacial baseada em autômatos celulares, foram gerados o mapa simulado e o cenário de prognóstico, considerando os projetos de expansão portuária para o ano de 2024. O crescimento das áreas construídas por influência dos projetos de expansão segue o sistema viário existente e a proximidade ao canal estuarino. Isso demonstra a tendência de localização de novas instalações em função das atividades portuárias, ligadas à circulação de mercadorias, e da infraestrutura implantada para o transporte de navegação e terrestre.

Palavras-chave: uso e cobertura da terra; autômatos celulares; região portuária; Porto de Santos; Baixada Santista.

\footnotetext{
Abstract:

Considering the pressures of the port infrastructure sector over the land use and physical planning in the region of Baixada Santista, such as the demand for backport areas as well as for
} 
loads storage and transport, this study aims to develop a dynamic model of landscape changes in this region and investigate the relationship between land cover change and the transport infrastructure in such port area. With the aid of remote sensing products and cellular automatabased modeling, a simulated landscape map and a map of future landscape changes scenario were generated, considering port expansion projects for the year 2024. The growth in built-up areas derived from expansion projects takes place along the existing road system and in the vicinities of the estuarine channel. This confirms the trend of new facilities allocation as a function of port activities related to the circulation of goods and the infrastructure associated with the sea and land transport.

Keywords: land cover; cellular automata; port region; Port of Santos; Baixada Santista.

\section{Introdução}

Cerca de dois terços da população mundial habitam as zonas costeiras, caracterizando um contingente populacional denso e concentrado, onde se localizam as principais metrópoles contemporâneas (Morais, 2007). A posição do espaço litorâneo favorece a circulação de produtos, detendo a primazia na alocação dos pontos de terminais dos fluxos intercontinentais de mercadorias, ainda hoje executados majoritariamente pelo transporte marítimo (Morais, 2007).

Dessa forma, os portos localizados em áreas costeiras geralmente desempenham atividades de impacto significativo no desenvolvimento socioeconômico das cidades portuárias. Mais do que estruturas que servem aos propósitos de circulação entre territórios, os portos também participam da produção do espaço, pelo fato de demandarem infraestruturas que possibilitem seu funcionamento nas mais variadas escalas geográficas (Machado, 2012).

Na região da Baixada Santista, além das estruturas ligadas ao Porto de Santos, as atividades referentes ao turismo de veraneio e à indústria do polo industrial de Cubatão marcaram, até recentemente, a estrutura econômica e urbanização da paisagem regional. Nos últimos anos, no entanto, a indústria extrativa ligada à extração de minerais não-metálicos voltados à atividade da construção civil regional e à exploração de petróleo e gás provenientes da Bacia de Santos tem recebido amplos investimentos (São Paulo, 2011).

Segundo a Codesp (2009), a projeção estimada de movimentação de cargas para o Porto de Santos deve alcançar aproximadamente 230 milhões de toneladas em 2024, sendo que para a viabilização desta meta, serão necessários investimentos da ordem de 7 bilhões de reais nos próximos anos, visando aumentar esta capacidade de movimentação, bem como modernizar as instalações e a infraestrutura do Porto de Santos.

Em vista da importância do Porto de Santos para a economia do estado de São Paulo e do país, da magnitude dos impactos na dinâmica de ocupação da região portuária e de seu entorno causados pela ampliação de suas instalações, assim como da repercussão desses impactos no ecossistema local, esta pesquisa objetiva a construção de modelos dinâmicos espaciais da paisagem para a região portuária da Baixada Santista, destinados a simular alterações pretéritas de uso e cobertura da terra no período de 2005 a 2013, visando ao entendimento das variáveis forçantes destas alterações em nível local. Com base nesta investigação, esta pesquisa propõe-se adicionalmente a elaborar cenários futuros dessas alterações, contemplando as propostas de expansão portuária previstas para o ano horizonte de projeto de 2024 , como forma de subsidiar o 
poder público na tomada de decisões frente às mudanças de uso e cobertura da terra esperadas para esta região, no que tange à gestão territorial e ambiental.

\section{Material e métodos}

\section{1 Área de estudo}

A Baixada Santista está localizada na região central do litoral do estado de São Paulo, e a área de estudo compreende os municípios de Santos, São Vicente, Praia Grande, Cubatão e Guarujá, apresentada na Figura 1. Esses municípios pertencem à Região Metropolitana da Baixada Santista (RMBS), juntamente com outros quatro: Bertioga, Peruíbe, Mongaguá e Itanhaém.

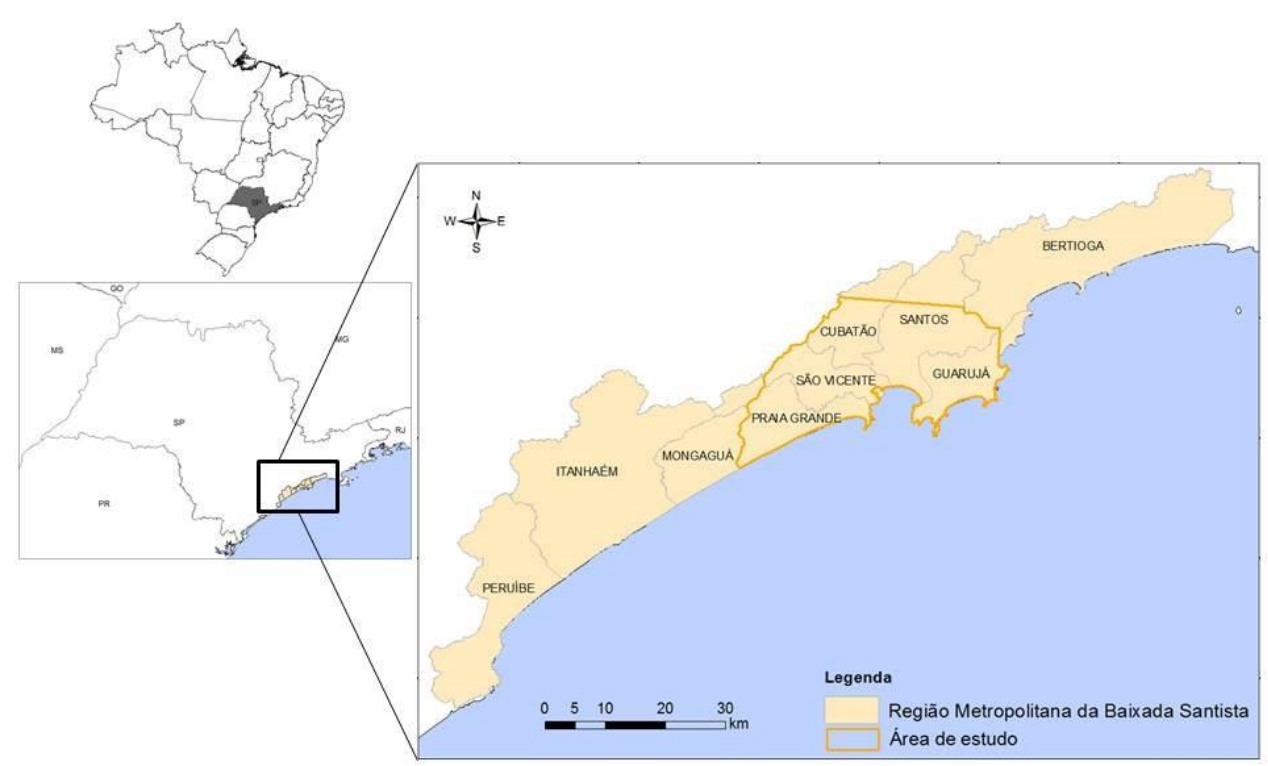

Figura 1: Localização da área de estudo na Região Metropolitana da Baixada Santista (RMBS).

Trata-se de uma região com uma complexa relação ambiental, populacional e de atividades econômicas, que reúne vocações aparentemente díspares, como o complexo portuário e industrial de grande porte e a vocação turística, que se mesclam às reservas ambientais e áreas de proteção legal (Zündt, 2006).

Em referência à área territorial do Porto de Santos, este ocupa parte dos municípios de Cubatão, Santos e Guarujá, estendendo-se ao longo do estuário e limitado pelas Ilhas de São Vicente e Santo Amaro. O sistema de infraestrutura de transportes é multimodal e permite a interligação do maior porto da América Latina com o interior do país, além dos países do MERCOSUL, interligados pelas rodovias e ferrovias (São Paulo, 2011).

Quanto ao sistema rodoviário, a RMBS é servida pelo complexo Anchieta-Imigrantes (SP-150 e SP-160, respectivamente), ligando-se com a Região Metropolitana de São Paulo (RMSP) e o interior do estado. Também faz conexão com as rodovias Manoel Hypólito do Rego (RioSantos/BR-101), ligação com o litoral norte e sul pelas rodovias Cônego Domênico Rangoni e Padre Manoel da Nóbrega (SP-055). No modo ferroviário, sua infraestrutura envolve a utilização 
direta das malhas da antiga Rede Ferroviária Federal S.A. (RFFSA), atualmente concessionada à empresa MRS Logística S.A., e da antiga Ferrovia Paulista S.A. (FEPASA), concessionada à empresa Ferrovia Bandeirantes (FERROBAN) (São Paulo, 2011).

A descoberta de petróleo no pré-sal da Bacia de Santos tem estimulado a expansão e desenvolvimento da região, além do crescimento da produção industrial ligada ao ramo da petroquímica e siderúrgica em Cubatão. Ademais, tem ocorrido o incremento das vias de acesso, tais como a segunda pista da Rodovia dos Imigrantes (SP-160), possibilitando maior atração populacional, em função da facilidade de deslocamento e desenvolvimento socioeconômico (São Paulo, 2011).

\subsection{Base de Dados}

Os dados utilizados na pesquisa constam da Tabela 1.

Tabela 1: Base de dados originais utilizados na pesquisa.

\begin{tabular}{cc}
\hline \multicolumn{1}{c}{ Base de Dados } & Fonte \\
\hline Altitude e Declividade & VALERIANO (2004) \\
\hline Unidades de conservação & MMA (2012) e SÃO PAULO (2006) \\
\hline Ferrovias & DER (2001) \\
\hline Hidrografia & DER (2001) \\
\hline Vias secundárias & BRASIL (2002) \\
\hline Poligonal & CODESP (2006, 2009) \\
\hline Novos empreendimentos & SÃO PAULO (2013) \\
\hline ZEE marinho e terrestre & DER (2001) \\
\hline Rodovias & CETESB (1991) \\
\hline Área industrial & CETESB (1991) \\
\hline Área urbana & Classificação automática a partir das imagens de satélite \\
Mapas de uso e cobertura da & Landsat INPE (2014), órbita-ponto 219/77 \\
terra (2005 e 2013)
\end{tabular}

Foram construídos mapas de distância às bases vetoriais das áreas urbanas, áreas industriais, poligonal da área portuária de Santos, base viária, atualizadas por período, bem como das rodovias e vias secundárias, ferrovias, hidrografia, áreas protegidas, empreendimentos operacionais e projetados. Esses mapas constituem-se em grades regulares no formato ASCII, com a resolução de $30 \mathrm{~m}$, em que a distância fornecida é a euclidiana, contada de centroide a centroide de cada célula da grade. Os sistemas viários foram atualizados com base em imagens auxiliares, do mesmo modo que as áreas industriais e urbanas.

Além dos mapas de distância, foram utilizadas as variáveis categóricas referentes às Unidades de Conservação, ao Zoneamento Ecológico-Econômico da Baixada Santista (ZEE-BS) Marinho e Terrestre, aos empreendimentos operacionais e projetados. 


\subsection{Mapas de uso e cobertura da terra}

A classificação das imagens de satélite visou a obtenção dos mapas de uso e cobertura da terra da região portuária da Baixada Santista, e a descrição de suas classes baseou-se nas proposições do projeto CORINE (Bossard et al., 2000), compreendendo, no caso particular deste trabalho, apenas as classes Urbano, Solo, Campo, Água, Floresta e Manguezal. As datas do imageamento foram 06/05/2005, com o satélite Landsat 5 TM, e 12/05/2013 com o satélite Landsat 8 OLI, bandas espectrais do azul, verde, vermelho, infravermelho próximo e médio.

Realizou-se o procedimento de tabulação cruzada entre os mapeamentos temáticos derivados das classificações das imagens TM de 2005 e OLI de 2013, a fim de identificar as transições de uso e cobertura da terra, sendo que o mapa mais recente (2013) foi ajustado considerando apenas as permanências e transições de degração, conforme Kawashima et al. (2015).

\subsection{Modelagem dinâmica espacial}

A modelagem dinâmica espacial presta-se a simular, em ambiente computacional, fenômenos do mundo real que possuem uma dimensão tanto espacial quanto temporal. Modelos dinâmicos espaciais envolvem uma ampla gama de aplicações, que compreendem a simulação de incêndios florestais, escoamento superficial e enchentes, expansão de epidemias, migração de espécies florestais, circulação de pedestres e veículos, mudanças de uso e cobertura da terra como expansão urbana, desmatamento, entre outras (Soares-Filho et al., 2002; Almeida et al., 2003; Almeida et al., 2005; Pinto e Antunes, 2007; Ximenes et al., 2008; Silvestrini et al., 2009; Macedo et al., 2013; Liu et al., 2015).

O paradigma matemático-computacional mais amplamente difundido para a construção de modelos dinâmicos espaciais consiste nos chamados autômatos celulares. Essa abordagem compreende uma grade de células regulares com $n$-dimensões, no qual o estado de cada célula depende de seu estado prévio e de um conjunto de regras de transição, de acordo com um arranjo específico de vizinhança, sendo todas as células atualizadas simultaneamente em passos de tempo discretos (Wolfram, 1984; Soares-Filho et al., 2007).

Os modelos dinâmicos de simulação de mudanças de uso e cobertura da terra tentam replicar possíveis padrões da evolução da paisagem e assim possibilitar a avaliação de futuras implicações ecológicas sobre o meio ambiente (Soares-Filho et al., 2002). De acordo com Soares-Filho et al. (2007), os componentes de um modelo de mudança da paisagem consistem em (1) uma configuração inicial, (2) função de mudança e (3) uma configuração de saída. Os autores descrevem a configuração inicial, como, por exemplo, um mapa da paisagem ou de uso e cobertura da terra, derivados de dados de sensoriamento remoto. A função de mudança pode ser contínua ou discreta, envolvendo desde uma equação linear diferencial, no caso de o tempo ser considerado como uma variável contínua, ou exemplos mais complexos, como a utilização de sequências de regras de decisão (Soares-Filho et al., 2007). A saída consiste em mapas simulados definidos em determinado intervalo de tempo.

A discretização de modelos de simulação passa pela seleção de variáveis explicativas e pelo cálculo de seus efeitos nas mudanças, resultando em um mapa de favorabilidade ou 
probabilidade de mudança (Soares-Filho et al., 2007). A seleção dessas variáveis determina as relações de influência com a variável dependente, definindo as células com maior ou menor probabilidade de transição de uso e cobertura da terra. As variáveis explicativas utilizadas na modelagem podem ser discriminadas em variáveis proximais e causais. As variáveis proximais explicam as influências locais na configuração espacial dos padrões de mudanças, como, por exemplo, distâncias às estradas e às cidades. As variáveis causais explicam as forças subjacentes ou motivadoras das mudanças, sendo muitas vezes consideradas como variáveis de contorno ou exógenas ao modelo espacial, a exemplo de modelos climáticos, demográficos, econômicos e político-econômicos e seus efeitos na dinâmica do sistema estudado (Soares-Filho et al., 2007).

Neste trabalho, utilizou-se a plataforma de modelagem baseada em autômatos celulares denominada Dinamica EGO 2.4, ferramenta livre desenvolvida pelo Centro de Sensoriamento Remoto da Universidade Federal de Minas Gerais, a qual permite a construção de modelos de simulação com o emprego de funções de transição concebidas para reproduzir as dimensões e as formas das alterações da paisagem (Soares-Filho et al., 2002). Uma série de procedimentos de conversão entre formatos dos mapas de variáveis e de paisagem foi necessária para a entrada desses dados no Dinamica EGO, os quais resultaram em arquivos matriciais estruturados na forma de um cubo de variáveis estáticas, apresentados na Figura 2. Esses procedimentos consistiram na conversão de arquivos GeoTIFF e ASCII para os formatos correspondentes no SIG IDRISI, e do formato IDRISI para o formato ERMapper, com extensão .ers.

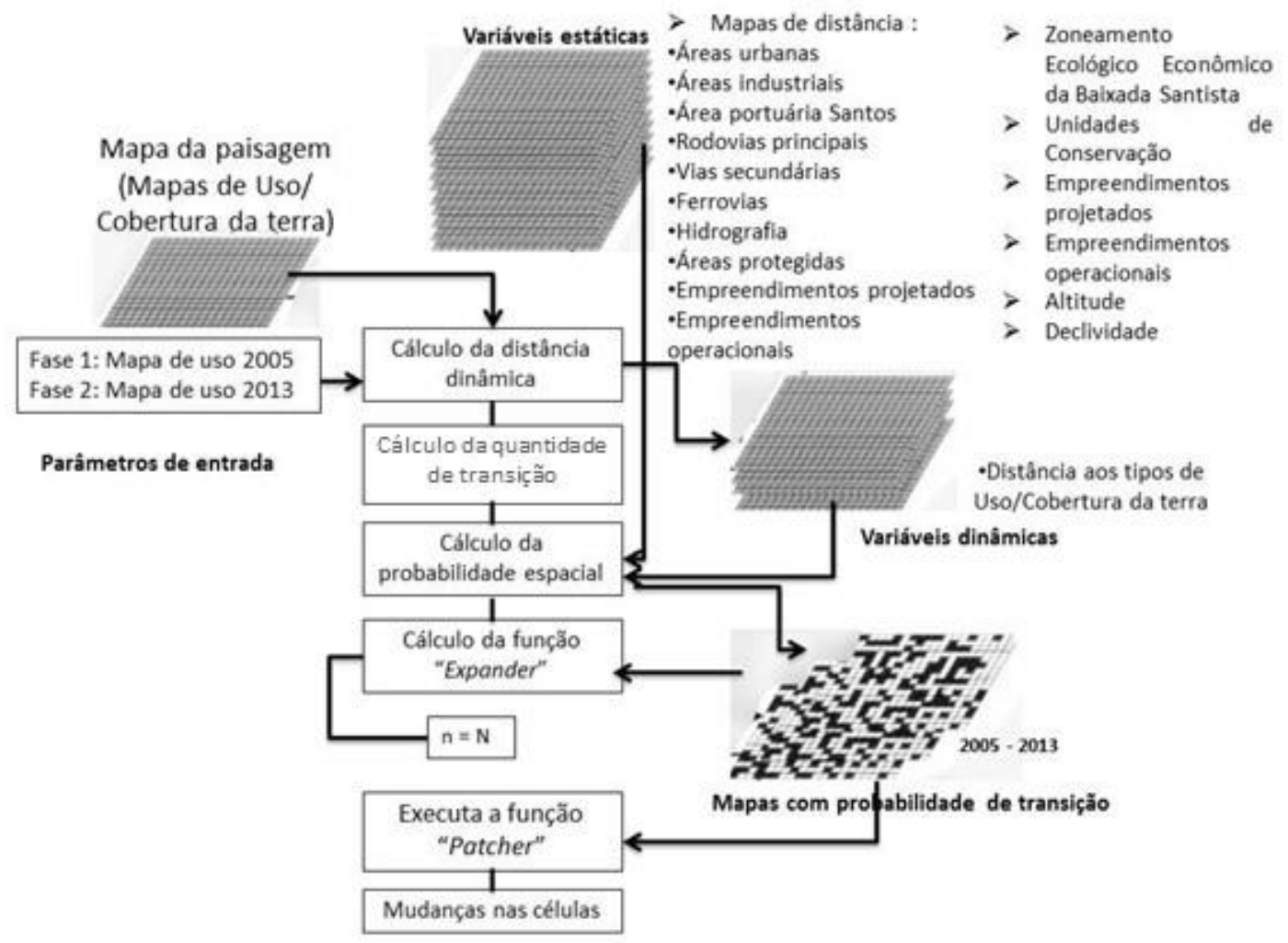

Figura 2: Base de dados e esquema descritivo da plataforma Dinâmica EGO. Fonte: Soares-Filho, Cerqueira e Pennachin (2002). Adaptado por Renata Sayuri

Kawashima, 2015.

As simulações foram realizadas com base nas taxas e probabilidades de transição das células, sendo que estas últimas foram determinadas pelo método de pesos de evidência. As saídas das simulações foram continuamente calibradas, e seus resultados validados.

As principais etapas seguidas para a construção da modelagem no Dinamica EGO foram: i) cálculo do percentual de mudanças das classes através das matrizes de transição; ii) cálculo das 
faixas de discretização das variáveis explicativas; iii) cálculo dos pesos de evidência $\left(\mathrm{W}^{+}\right)$, método bayesiano baseado na teoria da probabilidade condicional. Ele foi utilizado para o cálculo da probabilidade de mudança de estado (uso ou cobertura da terra) das células e representa a influência de cada categoria (ou faixa de valores) de certa variável espacial na probabilidade espacial de uma dada transição $i \rightarrow j$ para cada célula (Soares-Filho et al., 2007); iv) análise da associação ou dependência espacial entre as variáveis de entrada para todas as transições; v) simulação e calibração; vi) validação do mapa simulado, com base no mapa final observado, derivado da classificação da imagem OLI.

Para o cálculo do peso positivo de evidência, é utilizada uma equação que converte a fórmula de log odds ou logit (logaritmo neperiano do odds ou chance, dada pela divisão entre a probabilidade de ocorrência de um evento e a sua probabilidade complementar) para uma fórmula de probabilidade condicional comum, conforme Equação 1 e Equação 2 abaixo (Novaes, 2010):

$$
\begin{aligned}
& P\left(T_{i}^{\alpha} / V_{i}^{1}, \ldots, V_{i}^{m_{\alpha}}\right)=O\left(T_{i}^{\alpha}\right) \cdot e^{\sum_{v-1}^{m_{\alpha}} W^{+} i, y} / 1+O\left(T_{i}^{\alpha}\right) \cdot e^{\sum_{v-1}^{m_{\alpha}} W_{i, v}^{+}} \\
& \mathrm{W}^{+}=\log \mathrm{e} \frac{\mathrm{P}\left\{\mathrm{V}_{\mathrm{i}} / \mathrm{T}\right\}}{\mathrm{P}\left\{\mathrm{V}_{\mathrm{i}} / \overline{\mathrm{T}}\right\}}
\end{aligned}
$$

Em que:

$P$ corresponde à probabilidade de transição $T$ de uso ou cobertura da terra; $i$ corresponde a uma notação de posicionamento das células da área de estudo, definido em termos de coordenadas $x, y ; \alpha$ representa um tipo de transição de uso ou cobertura da terra, por exemplo, de uma classe $c$ para uma classe $k ; V i^{1}$ corresponde à primeira variável observada na célula $i$, utilizada para explicar a transição $\alpha ; V_{i}^{m \alpha}$ corresponde à $m$-ésima variável observada na célula $i$, utilizada para explicar a transição $\alpha ; O\left(T_{i}{ }^{\alpha}\right)$ representa o odds ou chance da transição $T^{\alpha}$ na $i$-ésima célula, expressa pela razão entre a probabilidade de ocorrência de $T_{i}{ }^{\alpha}$ e a probabilidade complementar, isto é, $P\left(T_{i}{ }^{\alpha}\right) / P\left(T_{i}{ }^{a}\right)$; e, $W^{+}{ }_{i, v}$ corresponde ao peso positivo de evidência para a $i$-ésima célula referente à $v$-ésima variável.

Assim, a partir dos mapas de uso e cobertura da terra (anos de 2005 e 2013) e do cubo de dados, foram calculados os pesos de evidência para todas as variáveis e respectivos intervalos categóricos em todas as transições.

A dependência espacial entre pares de variáveis foi avaliada através do método da Incerteza de Informação Conjunta (Joint Information Uncertainty), implementada no Dinamica EGO. Valores superiores a 0,5 indicam associação significativa entre pares de variáveis (Bonham-Carter, 1994). Caso esse valor ocorra, uma das variáveis deve ser excluída do modelo, ou ambas combinadas em um único plano de informação para evitar viés no modelo (Bonham-Carter, 1994).

As simulações foram realizadas a partir da calibração do modelo, que envolveu a definição dos percentuais de contribuição dos algoritmos expander e patcher, em que o expander dedica-se unicamente à expansão ou contração de manchas de uma determinada classe, e a função patcher é responsável por formar novas manchas (Soares-Filho et al., 2007). A calibração também compreendeu a definição dos valores de tamanho médio e variância do tamanho de manchas para cada transição de classes, sendo que os valores de índice de isometria das manchas foram definidos empiricamente, considerando o padrão de distribuição espacial das novas manchas. Segundo Ximenes et al. (2008), este índice corresponde a um valor entre 0 e 2, que é multiplicado pelo valor de probabilidade das oito células da janela de vizinhança $3 \times 3$, utilizada 
pelos algoritmos expander e patcher, antes da aplicação da regra de transição. Quanto maior o valor adotado, mais geometricamente regulares serão as manchas produzidas pelos algoritmos de transição, e, de forma contrária, quanto mais próximo de zero, mais dendríticas serão as manchas.

A validação das simulações foi realizada em função da incerteza da localização de uma classe dentro de sua vizinhança de células, conhecida como fuzziness of location, método proposto por Hagen (2003), considerando janelas de vizinhança de 3x3 a 11x11 pixels e comparando-se os mapas-diferença. Os dois mapas-diferença empregados na validação são gerados, por um lado, a partir de uma subtração, a qual mascara as áreas de não-mudança, entre o mapa de uso e cobertura final real e o mapa de uso e cobertura inicial, e por outro lado, entre o mapa de uso e cobertura final simulado e o mapa de uso e cobertura inicial. Esse método pode empregar tanto uma função constante quanto uma função de decaimento, dada por $2^{-d / 2}$, obtida a partir do aumento da distância $d$, calculada de centroide a centroide, da célula central do mapa-diferença 1, utilizado como referência, até a célula contendo a classe esperada dentro da janela de vizinhança, posicionada sobre o mesmo local no mapa-diferença 2, utilizado para comparação.

\subsection{Construção do cenário de prognóstico}

Cenários são imagens alternativas de futuro que facilitam a tomada de decisões. Eles delineiam

conjeturas político-econômicas, sociodemográficas, legais, institucionais, ambientais, tecnológicas e outras, que serão responsáveis por desencadear mudanças passíveis de previsão, segundo um grau de certeza, em horizontes de projeto específicos. Cenários subdividem-se em estacionários e não-estacionários, em que os primeiros utilizam o diagnóstico das alterações pretéritas para direcionar as alterações que ocorrerão no futuro, reproduzindo tendências observadas no passado. Os não-estacionários, por sua vez, podem utilizar o diagnóstico das alterações pretéritas para simular alterações futuras, mas incorporam novas condições de contorno, como mudanças na conjuntura macro ou microeconômica, alterações em dispositivos legais, novas instalações de infraestrutura etc.

No caso particular deste trabalho, foi gerado um cenário não-estacionário considerando a implantação de empreendimentos portuários, cuja entrada em operação é prevista para ocorrer até o ano de 2024. Para a sua elaboração, foram mantidos os parâmetros obtidos no modelo de simulação gerado para o período de 2005 a 2013, no que tange ao tamanho médio e variância das manchas de paisagem, percentual de expander e patcher e índice de isometria. Uma nova matriz de transição foi extraída para o período entre 2013 e 2024, com o objetivo de quantificar o número total de novas células que transicionarão para a classe Urbano nesse horizonte de projeto.

\section{Resultados e discussão}

\subsection{Classificação das imagens de satélite}

O resultado da classificação da imagem para o ano de 2005 obteve exatidão global de $84 \%$ e índice Kappa de 0,81. A menor acurácia do usuário foi a da classe Solo, com 42\%, apresentando 
confusão, principalmente, com as classes Urbano e Água. A classe Manguezal apresentou confusão com a classe Floresta, obtendo acurácia de $86 \%$.

O resultado da classificação da imagem do ano de 2013 obteve exatidão global de $80 \%$ e o índice Kappa calculado foi de 0,76. A acurácia do usuário da classe Solo foi de apenas 38\%, principalmente em decorrência de pixels das classes de Água e Campo erroneamente classificados naquela classe.

Com a classificação das imagens e posteriores correções feitas com o auxílio de tabulação cruzada (álgebra de mapas), obtiveram-se os mapeamentos definitivos de uso e cobertura da terra para 2005 e 2013, apresentados na Figura 3, conforme Kawashima et al. (2015).
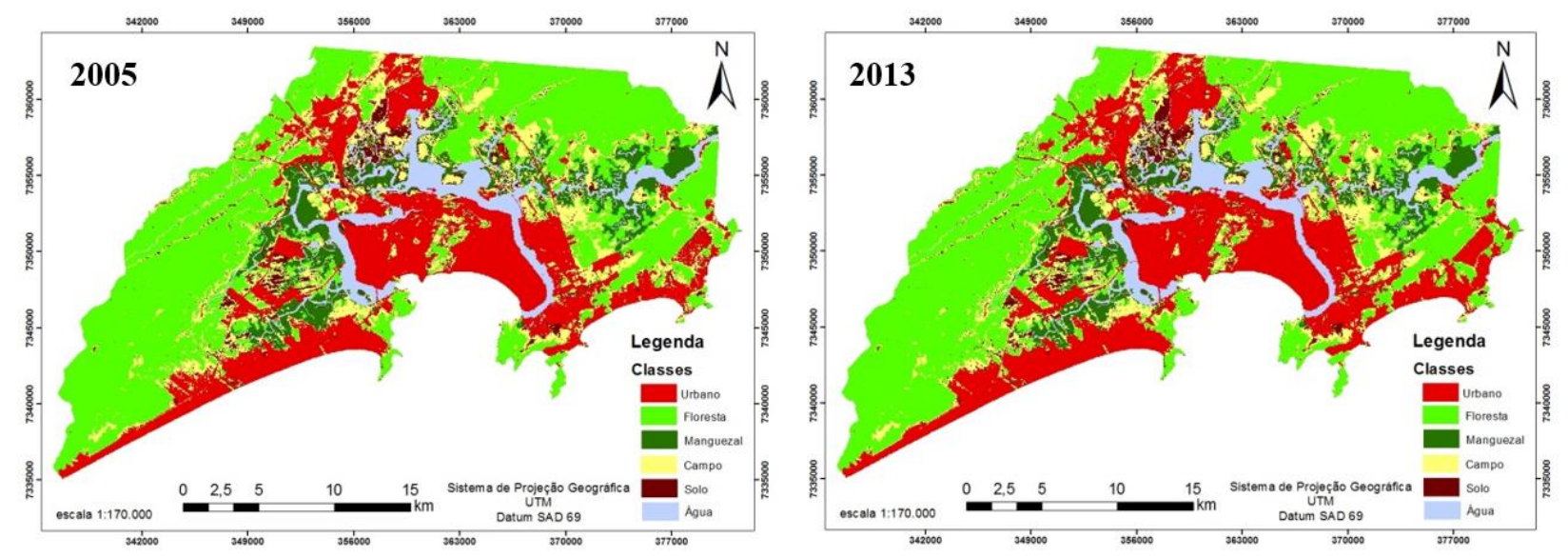

Figura 3: Mapas de uso e cobertura da terra de 2005 e 2013 da região portuária da Baixada Santista.

\subsection{Modelagem dinâmica espacial}

A Tabela 2 representa a matriz de transição para o período entre 2005 e 2013 em termos de áreas totais transicionadas entre as classes de uso e cobertura da terra.

Tabela 2: Matriz de transição de classes dada em área (ha) no período entre 2005 e 2013.

\begin{tabular}{ccccc}
\hline $\begin{array}{c}\text { Período: } \\
\text { 2005-2013 }\end{array}$ & \multicolumn{4}{c}{$\begin{array}{c}\text { Matriz de transição } \\
\text { (área em hectares) }\end{array}$} \\
\hline De/Para & Urbano & Campo & Solo & Total \\
\hline Floresta & 54,36 & 346,14 & 102,06 & 502,56 \\
Manguezal & 20,43 & 115,47 & 41,76 & 177,66 \\
Campo & 972,72 & - & 424,44 & 1397,16 \\
Solo & 328,5 & - & - & 328,5 \\
Água & 190,08 & - & - & 190,08 \\
Total & 1566,09 & 461,61 & 568,26 & 2595,96 \\
\hline
\end{tabular}

De acordo com a Tabela 2, as áreas da classe Floresta reduziram em 502,56 ha entre 2005 e 2013, dos quais 54,36 ha foram acrescidos para a classe Urbano, 346,14 ha para o incremento da classe Campo, e 102,06 ha para a classe Solo. As áreas de Manguezal foram reduzidas em 177,66 ha entre 2005 e 2013, convertendo-se para a classe Urbano em 20,43ha, 115,47 ha para a classe Campo, e 41,76 ha para a classe Solo. 
As áreas da classe Campo foram alteradas em 1.397,16 ha durante esse período, convertidas em 972,72 ha para a classe Urbano, e 424,44 ha para a classe Solo. As áreas florestadas apresentam uma tendência em se converterem em áreas degradadas, representadas pelas classes Campo e Solo, em um primeiro momento e, em uma segunda etapa, se transformarem na classe Urbano. A degradação da terra na área de estudo está relacionada à conversão para as classes Urbano, Campo e Solo. Não foi analisada a restauração florestal da região de estudo, uma vez que não houve análise da conversão dessas classes para Floresta ou Manguezal.

A transformação da classe Solo para Urbano foi de 328,5 ha, e a conversão da classe Água para Urbano foi de 190 ha, representando as novas instalações portuárias construídas no estuário durante esse período.

Os valores de pesos de evidência positivos referem-se à associação positiva entre a evidência e o evento, ou seja, favorecem a mudança de estado; já valores negativos indicam a repulsão causada pela evidência (variável) para a ocorrência do fenômeno (evento de mudança), e valores próximos de zero significam que a variável não exerce efeito sobre a transição (Soares-Filho et al., 2009).

A transição de áreas florestais para urbanas apresentou influência positiva com a maior proximidade à hidrografia em até $300 \mathrm{~m}$ de distância, apresentado na Figura 4. Teixeira et al. (2009) verificaram que a distância à hidrografia favoreceu a conversão da Floresta Atlântica em Ibiúna, SP, para áreas ocupadas em locais mais distantes dos rios, provavelmente porque esses rios não são navegáveis e a mata ciliar tem sido protegida pela legislação ambiental. Por outro lado, a ocupação do Porto de Santos próxima ao estuário é um importante fator para o desenvolvimento da região.

De acordo com a Figura 4, houve influência das vias secundárias mais intensamente na faixa de distância até 200 m entre 2005 e 2013, com influência na transição para novas áreas construídas. A variável dinâmica de distância à classe Campo indica que a transição foi influenciada positivamente na faixa de distância entre 120 e $150 \mathrm{~m}$. Em relação à variável categórica referente às Unidades de Conservação (UCs), o valor dos pesos de evidência apresentou-se negativo, dado que as áreas protegidas inibem esse tipo de transição.
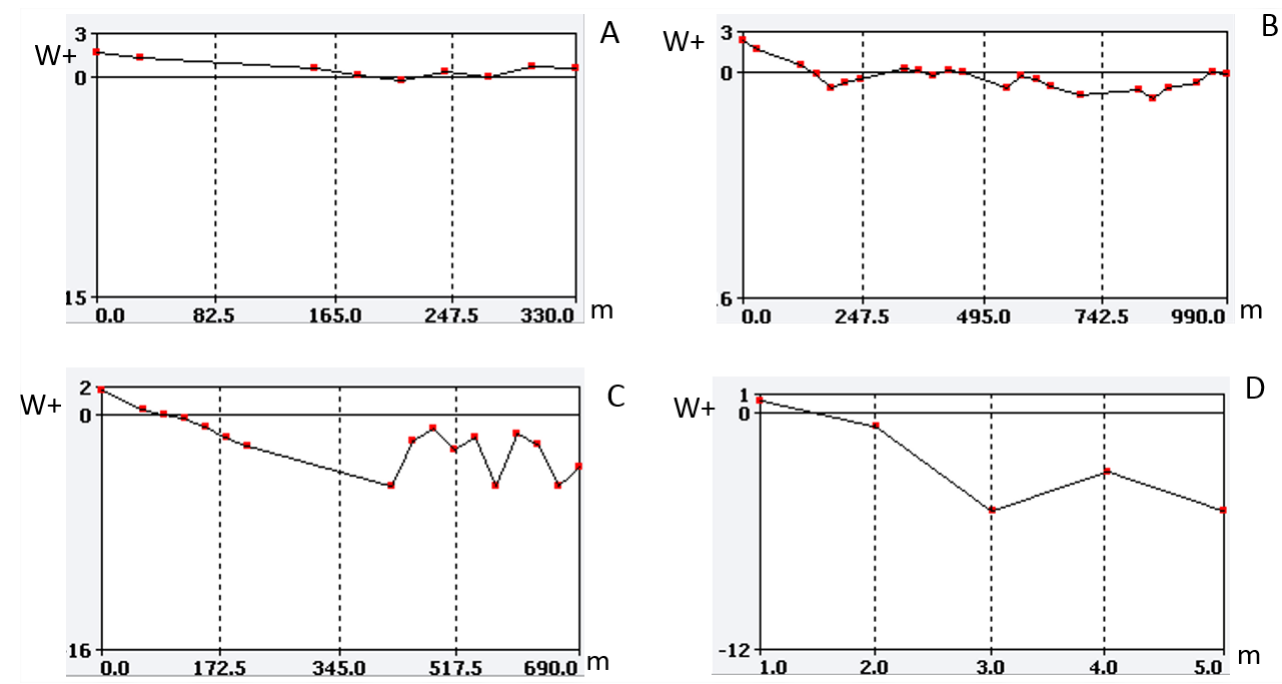

Figura 4: Valores de pesos de evidência em função das variáveis explicativas relativos à transição Floresta para Urbano. $\mathrm{A}=$ Distância à hidrografia; $\mathrm{B}=$ Distância às vias secundárias; $\mathrm{C}=$ Distância à classe Campo; D = Distância às Unidades de Conservação (UCs).

As rodovias influenciaram na transição da classe Manguezal para Urbano em distâncias até 500 $\mathrm{m}$, e as vias secundárias entre 300 e $400 \mathrm{~m}$. 
Cunha-Lignon et al. (2009) analisaram os impactos antrópicos ocorridos em áreas de manguezal no estado de São Paulo e descobriram que nos manguezais da Baixada Santista, especialmente na Ilha Barnabé, a construção de rodovia e ferrovia, a expansão portuária e a retificação de canal podem ter provocado a perda de extensas áreas de manguezal por aterros, alagamentos, cortes de vegetação e alterações da hidrodinâmica local.

Com relação à transição da classe Água para Urbano, a variável representada pelas vias secundárias apresentou influência nessa transição em distâncias até $200 \mathrm{~m}$, e em distâncias até $150 \mathrm{~m}$ da própria classe Urbano. Os novos empreendimentos operacionais, representados pelo Terminal da Embraport e o Brasil Terminal Portuário (BTP), apresentaram valores positivos de pesos de evidência e foram determinantes na transição da classe Água para Urbano em distâncias até $300 \mathrm{~m}$.

Com relação a esses novos empreendimentos, em novembro de 2009, o BTP iniciou o processo de remediação ambiental sobre o antigo lixão na área da Alemoa, com resíduos provenientes principalmente do porto. Seus trabalhos foram concluídos em março de 2012, com investimentos de quase dois bilhões de reais, e sua operação comercial se iniciou em agosto de 2013 (Btp, 2014).

Com relação ao Terminal da Embraport, Empresa Brasileira de Terminais Portuários, este foi construído na margem esquerda do estuário com aterro de áreas de manguezais. Sua movimentação está prevista em aproximadamente 1,2 milhão de contêineres e dois milhões de $\mathrm{m}^{3}$ de álcool por ano (Scazufca, 2012). Em 2007, iniciaram-se as obras com a construção de um aterro-piloto para estudo do solo da região, e em 2010, as obras se intensificaram, finalizando-se em 2013, tendo sido o início das operações em julho do mesmo ano com a chegada do primeiro navio, o MERCOSUL Manaus (Embraport, 2014).

O aumento da atividade portuária em Santos, ou mesmo a modernização visando maior pordutividade, implicam novos territórios operacionais, localizados primeiramente no canal de navegação do porto, próximos às áreas urbanizadas, ocupadas pelas cidades que abrigam a região portuária, e também áreas que ainda conservam alguma cobertura vegetal, geralmente manguezais (Scazufca, 2012).

A avaliação do mapa simulado para o ano de 2013 é apresentada na Tabela 3, e o resultado da simulação na Figura 5.

Tabela 3: Avaliação do resultado do modelo de simulação para o ano de 2013.

\begin{tabular}{ccccc}
\hline $\begin{array}{c}\text { Tamanho da } \\
\text { Janela (pixels) }\end{array}$ & $\begin{array}{c}\text { Decaimento exponencial } \\
\text { Mínima }\end{array}$ & $\begin{array}{c}\text { Máxima } \\
\text { Similaridade }\end{array}$ & $\begin{array}{c}\text { Decaimento constante } \\
\text { Similarima } \\
\text { Similaridade }\end{array}$ & $\begin{array}{c}\text { Máxima } \\
\text { Similaridade }\end{array}$ \\
\hline $3 \times 3$ & 0,436 & 0,456 & 0,483 & 0,510 \\
$5 \times 5$ & 0,485 & 0,504 & 0,590 & 0,616 \\
$7 \times 7$ & 0,509 & 0,527 & 0,669 & 0,688 \\
$9 \times 9$ & 0,521 & 0,538 & 0,727 & 0,743 \\
$11 \times 11$ & 0,528 & 0,544 & 0,775 & 0,782 \\
\hline
\end{tabular}

No referido mapa simulado para 2013, apresentado na Figura 5, o arquivo de pesos de evidência com os novos empreendimentos operacionais entra em execução na plataforma Dinamica EGO somente a partir do sexto ano da simulação, ou seja, a partir de 2010, sendo que o último ano é o nono da simulação. O mapa simulado do ano de 2013 não foi capaz de prever todas as novas instalações portuárias finalizadas naquele ano. Uma alternativa para solucionar esse problema seria a insersão de uma nova matriz de transição, intensificando a transição da classe Água para a classe Urbano, no período entre 2010 e 2013. 

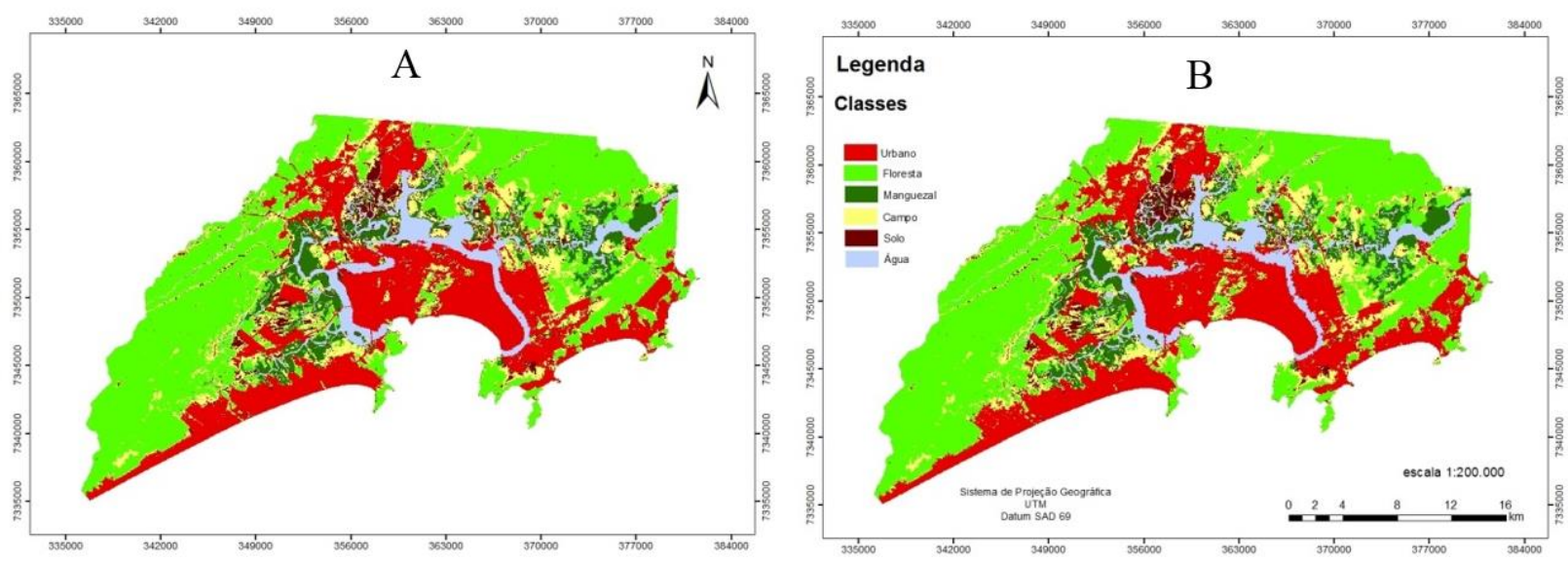

Figura 5: Resultado do mapa classificado de uso e cobertura da terra de 2013(A) e resultado do modelo simulado de mudanças de uso e cobertura da terra na região portuária da Baixada Santista para 2013(B).

\subsection{Modelo de prognóstico}

A nova matriz de transição de múltiplos passos, que fornece as taxas de transição de uso e cobertura da terra ano a ano para o período entre 2013 e 2024, é apresentada na Tabela 4.

Tabela 4: Matriz de transição de múltiplos passos no período entre 2013 e 2024.

\begin{tabular}{llll}
\hline Período: 2013-2024 & \multicolumn{3}{c}{ Matriz de transição de múltiplo passo (percentuais) } \\
\hline De/Para & Urbano & Campo & Solo \\
\hline Floresta & $0,14 \%$ & $0,16 \%$ & $0,04 \%$ \\
Manguezal & $0,26 \%$ & $0,30 \%$ & $0,10 \%$ \\
Campo & $1,23 \%$ & - & $0,60 \%$ \\
Solo & $2,19 \%$ & - & - \\
Água & $0,17 \%$ & - & - \\
\hline
\end{tabular}

De acordo com a Tabela 4, as classes Solo e Campo são as principais responsáveis por contribuírem para o aumento da classe Urbano, em $2,19 \%$ e $1,23 \%$, respectivamente, em cada passo de tempo.

Avaliando os valores acrescidos e reduzidos na projeção de 2024, foi possível observar que entre os anos de 2005 e 2024, a classe Urbano aumentou em 23,66\%, e entre 2013 e 2024, esse aumento representou 14,62\%. Já a classe Solo entre 2005 e 2024 cresceu em 9,8\%, e entre 2013 e 2024, aumentou em 1,56\%. Para as demais classes, houve redução, a exemplo da classe Campo, que entre 2005 e 2024 diminuiu em 22,05\%, e entre 2013 e 2024 reduziu em 11,8\% e a classe Floresta que reduziu entre 2005 e 2024, em 5,5\%, e entre 2013 e 2024, em 3,73\%. A classe Manguezal também reduziu entre os anos entre 2005 e 2024 em 10,46\%, e entre 2013 e 2024, redução em 6,9\%.

A simulação do cenário para o ano de 2024, com os novos empreendimentos projetados, é apresentada na Figura 6. 

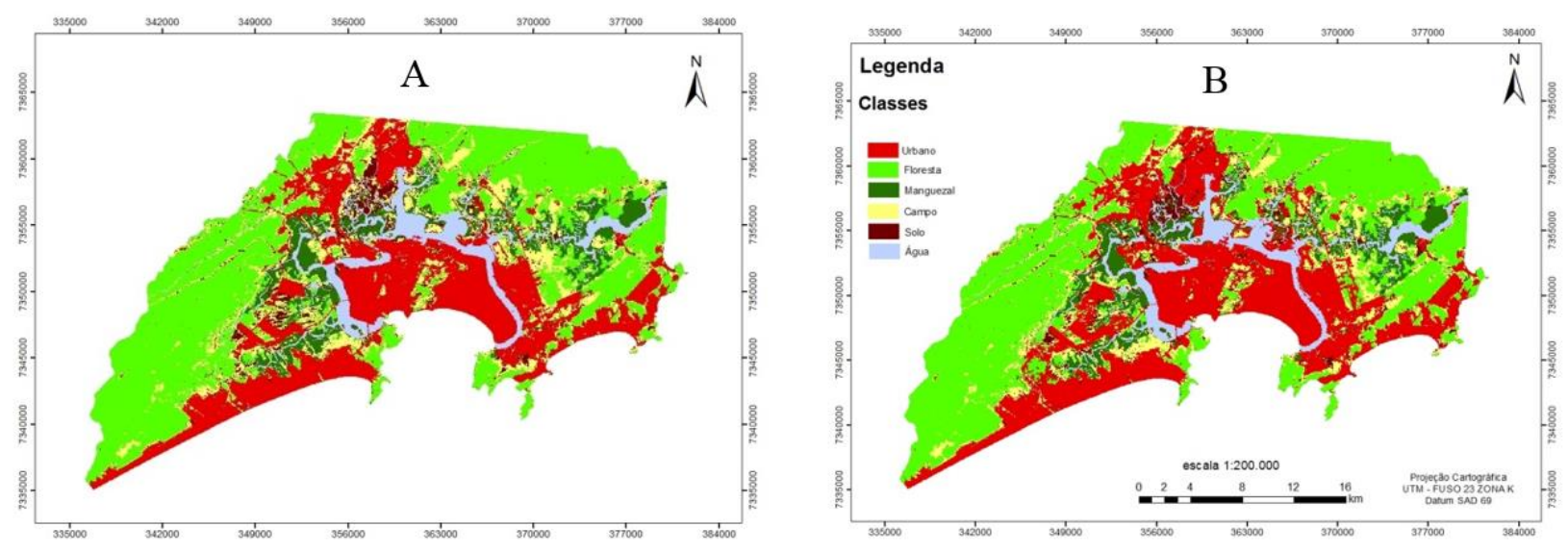

Figura 6: Resultado do mapa de uso e cobertura da terra de 2013 (A) e resultado do modelo simulado de mudanças de uso e cobertura da terra na região portuária da Baixada Santista, considerando novos empreendimentos projetados para o ano de 2024 (B).

É possível verificar a ocupação de áreas construídas devido à instalação de novos empreendimentos, principalmente aquelas próximas à região estuarina e na porção continental de Santos, Guarujá, Cubatão e São Vicente, ocorrendo nas proximidades das principais vias de acesso, como as rodovias.

Esse modelo simulado apresentou áreas com potencial para atração de novos empreendimentos, alguns verificados em processo de licenciamento ambiental, e estão relacionados com as futuras tendências para a região portuária de Santos nos próximos anos. Essas áreas correspondem àquelas que abrigam a classe Urbano e se encontram localizadas nas adjacências das áreas com instalações portuárias no ano de 2013. Além dos terminais de petróleo projetados para a região, são destaques também as instalações dos terminais retroportuários, os quais apresentam importante papel diante da conteinerização das mercadorias.

Como salientado por Scazufca (2012), desenvolvem-se nessas áreas atividades logísticas do transporte portuário, como depósito de contêineres ou de mercadorias, estoque, atividades de fiscalização e controle, além de pequenas operações de beneficiamento das mercadorias, viabilizando (devido à falta de áreas lindeiras aos cais) a movimentação dos terminais de contêineres.

Também por não estarem restritos quanto aos requisitos locacionais, já que não necessitam se situar justapostos ao canal de navegação, mas somente próximos à operação portuária, ou mesmo distantes da mesma desde que com fácil acesso, estes terminais têm se espalhado nas áreas urbanas ou junto a estas em grande parte do território dos municípios da parte central da Baixada, juntamente com o arco viário metropolitano (Scazufca, 2012).

\section{Conclusões}

Com relação à modelagem considerando os projetos de expansão do porto, é possível observar no mapa simulado as principais tendências de ocupação das áreas construídas, muitas vezes em regiões ocupadas em áreas de manguezal, representando a sua fragilidade frente à implantação dos novos empreendimentos. A modelagem dinâmica espacial permite a análise espacial das mudanças no uso e cobertura da terra, observando as conversões das áreas florestadas em áreas 
de degradação, representadas pelas classes Campo, Solo e Urbano, além de verificar as principais influências para essas conversões, tais como a presença de vias secundárias e da importância estratégica da hidrografia local, representando os canais de escoamento de mercadorias do Porto de Santos. Cabe notar as recém áreas de retroporto, para armazenamento e apoio logístico do Porto, verificados nos projetos de expansão portuária para os próximos anos.

Dessa forma, o trabalho mostra-se propício a fornecer diretrizes para o monitoramento dessas áreas através do desenvolvimento de modelos de simulação, com base na geração de cenários por meio de modelagem dinâmica espacial. A concepção de cenários distintos pode e deve ser utilizada como indícios da tendência de ocupação da região, e o mapa simulado deve ser desenvolvido preferencialmente de forma a integrar os distintos planos de gestão territorial.

\section{AGRADECIMENTOS}

Ao Marco Nalon, do Instituto Florestal, à Prof. ${ }^{a}$ Dr. ${ }^{a}$ Paulina Setti Riedel, do Instituto de Geociências da Unesp, e ao Prof. ${ }^{\circ}$ Dr. Luis Americo Conti, EACH-USP, pelas imagens de satélite fornecidas. À Dr. ${ }^{a}$ Ana Lucia Gomes dos Santos, pelas fotografias aéreas fornecidas.

\section{REFERÊNCIAS BIBLIOGRÁFICAS}

Almeida, C. M., Batty, M., Monteiro, A. M. V., Câmara, G., Soares-Filho, B. S., Cerqueira, G. C., and Pennachin, C. L. "Stochastic cellular automata modelling of urban land use dynamics: empirical development and estimation." Computers, Environment and Urban Systems 27 (2003): 481-509.

Almeida, C. M., Batty, M., Monteiro, M. V., Câmara, G., Soares-Filho, B. S., Cerqueira, G. C., and Pennachin, C. L. "GIS and remote sensing as tools for the simulation of urban land-use change.” International Journal of Remote Sensing 26 (2005): 759-774.

Bonham-Carter, G. "Geographic information systems for geoscientists: modelling with GIS". New York: Pergamon, 1994.

Bossard, M., Feranec, J., and Otahel, J. "CORINE land cover technical guide”. Addendum 2000. Technical report 40 (2000). EEA. Accessed November 9, 2014. http://www.eea.europa.eu/publications/tech40add.

Brasil. Decreto $n^{\circ} 4333$, de 12 de Agosto de 2002 - Regulamenta a delimitação das áreas do Porto Organizado de Fortaleza, Santos e Vitória, suas instalações, infraestrutura e planta $\begin{array}{llll}\text { geográfica. Accedded } & \text { August } & 013 .\end{array}$ http://www.planalto.gov.br/ccivil_03/decreto/2002/D4333.htm.

Btp. Brasil Terminal Portuário. Accessed October 04, 2014. http://www.btp.com.br/en/conhecaa-btp/quem-somos/.

Cetesb. Companhia de Tecnologia de Saneamento Ambiental. "Avaliação do estado de degradação dos ecossistemas da Baixada Santista”. São Paulo: CETESB, 1991, 45p.

Codesp. Companhia Docas do Estado de São Paulo. "Plano de Desenvolvimento e Zoneamento do Porto de Santos PDZ 2006." Accessed November 02, 2013. http://www.portodesantos.com.br/pdzps.php

Codesp. Companhia Docas do Estado de São Paulo. "Plano de Desenvolvimento e Expansão do Porto de Santos PDEPS 2009." Accessed June 05, 2014. 
http://www.portosdobrasil.gov.br/assuntos-1/relacoes-internacionais/arquivos/relatorio-finalplano-diretor-bid.pdf>.

Cunha-Lignon, M., Menghini, R. P., Santos, L.C.M., Niemeyer-Dinóla, C., and SchaefferNovelli, Y. "Estudos de Caso nos Manguezais do Estado de São Paulo (Brasil): Aplicação de Ferramentas com Diferentes Escalas Espaço-Temporais". Revista de Gestão Costeira Integrada, 9 (2009): 79-91.

Der. Departamento de Estradas de Rodagem. “Base de dados do DER”. São Paulo, 2001.

Embraport. Empresa Brasileira de Terminais Portuários. Accessed October 04, 2014. http://www.terminalembraport.com.br/a-embraport/historia.

Hagen, A. "Fuzzy set approach to assessing similarity of categorical maps." International Journal of Geographical Science 17 (2003): 235-249.

Ibge. Instituto Brasileiro de Geografia e Estatística. "Bases e referenciais." 1985. Accessed February 13, 2014. http://mapas.ibge.gov.br/bases-e-referenciais/bases-cartograficas/cartas.

Inpe. Instituto Nacional de Pesquisas Espaciais. “Catálogo de imagens”. São José dos Campos, SP. Accessed February 10, 2014. http://www.dgi.inpe.br/CDSR/.

Kawashima, R. S., Almeida, C. M., Giannotti, M. A., and Quintanilha, J. A. "Análise das mudanças temporais de cobertura da terra na região portuária da Baixada Santista-SP e a proposição de modelos de dinâmica espacial." In Anais XVII Simpósio Brasileiro de Sensoriamento Remoto, João Pessoa- PB, April, 1082-1089, 2015.

Kawashima, R. S. Modelagem da dinâmica espacial das mudanças na cobertura da terra na região portuária da Baixada Santista. Dissertação de Mestrado. Universidade de São Paulo, São Paulo, 190 p, 2015.

Liu, L., Liu, Y., Wang, X., Yu, D., Liu, K., Huang, H., and Hu, G. "Developing an effective 2-D urban flood inundation model for city emergency management based on cellular automata." Natural Hazards and Earth System Sciences 15 (2015): 381-391.

Macedo, R. C., Almeida, C. M., Santos, J. R., and Rudorff, B. F. T. "Modelagem dinâmica espacial das alterações de cobertura e uso da terra relacionadas à expansão canavieira." Boletim de Ciências Geodésicas 19 (2013): 313-337.

Machado, I. S. "O Porto de Santos e a Revolução dos Contêineres”. Dissertação (Mestrado) Faculdade de Filosofia, Letras e Ciências Humanas, Universidade de São Paulo, São Paulo, SP. 165p. 2012

Mma. Ministério do Meio Ambiente. 2012. "I3geo." Accessed April 05 abr, 2013. http://mapas.mma.gov.br/i3geo/datadownload.htm.

Morais, A. C. R. "Contribuições para a gestão da zona costeira do Brasil: elementos para uma geografia do Litoral Brasileiro”. 2ed. São Paulo: Annablume, 232p, 2007.

Novaes, M. R. "Análise da redução da queima na colheita da cana-de-açucar por meio de modelagem dinâmica espacial." Dissertação (Mestrado) - Instituto Nacional de Pesquisas Espaciais, São José dos Campos, 155p, 2010.

Pinto, N. N., and Antunes, A. P. "Cellular automata and urban studies: a literature survey." Architecture, City and Environment 1 (2007): 368-399.

São Paulo. Secretaria de Estado do Meio Ambiente. "Áreas especialmente protegidas." Secretaria de Estado do Meio Ambiente, Coordenadoria de Planejamento Ambiental Estratégico 
e Educação Ambiental, texto José Flávio de Oliveira; Ana Fernandes Xavier; Hiede Luciana dos Santos, São Paulo: SMA/CPLEA, 2006. Coletânea de Leis e Mapas 1 CD-ROM.

São Paulo. Secretaria de Estado do Meio Ambiente. "Zoneamento Ecológico- Econômico da Baixada Santista." 2013. Accessed 17 April,2014.http://www.ambiente.sp.gov.br/cpla/zoneamento/zoneamento-ecologicoeconomico/baixada-santista.

São Paulo. Secretaria de Planejamento e desenvolvimento Regional. "Caracterização socioeconômica de São Paulo - Região Metropolitana da Baixada Santista." 2011. Accessed 05 February, 2014.http://www.planejamento.sp.gov.br/noti_anexo/files/uam/trabalhos/RMBS.pdf>

Scazufca, M. “A primazia do Porto de Santos no cenário portuário nacional no período contemporâneo- Determinantes logísticos, territoriais e de gestão". Tese (Doutorado)Faculdade de Arquitetura e Urbanismo,Universidade de São Paulo, São Paulo, 385 p, 2012.

Silvestrini, R., Soares-Filho, B. S., Alencar, A. A. C., Rodrigues, H. O., Assunção, R. M., and Mendonza, E. "Modelo probabilístico de espalhamento de fogo: Aplicação para a região do Xingu". Anais XIV Simpósio Brasileiro de Sensoriamento Remoto, Natal - RN, April, 54595466, 2009.

Soares-Filho, B. S., Cerqueira, G. C., and Pennachin, C. L. "DINAMICA- A stochastic cellular automata model designed to simulate the landscape dynamics in an Amazonian colonization frontier." Ecological Modelling 154 (2002): 217- 235.

Soares-Filho, B. S., Cerqueira, G. C., Araújo, W. L., and Voll, E. "Modelagem de dinâmica de paisagem: concepção e potencial de aplicação de modelos de simulação baseados em autômato celular." Megadiversidade 3 (2007): 74-86.

Soares-Filho, B. S., Rodrigues, H. O., and Costa, W. L. Trad. Lima, S. "Modelagem de dinâmica Ambiental com DINAMICA EGO". Centro de Sensoriamento Remoto. 2009. Universidade Federal de Minas Gerais, Belo Horizonte, MG. Accessed 20 June, 2013. http://www.csr.ufmg.br/dinamica.

Teixeira, A. M. G., Soares-Filho, B. S., Freitas, S. R., and Metzer, J. P. "Modeling landscape dynamics in na Atlantic Rainforest region: implications for conservation". Forest Ecology and Management, v. 257, n.4 (2009): 1219-1230.

Valeriano, M. M. "Modelo digital de elevação com dados SRTM disponíveis para a América do Sul." São José dos Campos, SP: INPE: Coordenação de Ensino, Documentação e Programas Especiais (INPE-10550-RPQ/756), 2004, 72p.

Wolfram, S. "Universality and complexity in cellular automata." Physica 10D (1984): 1-35. 1984.

Ximenes, A. C. , Almeida, C. M., Amaral, S., Escada, M. I. S., and Aguiar, A.P. D. "Modelagem Dinâmica do Desmatamento na Amazônia." Boletim de Ciências Geodésicas 14 (2008): 370391.

Zündt, C. "Baixada Santista: uso, expansão e ocupação do solo, estruturação de rede urbana regional e metropolização”. In: Cunha, J. M. P. (Org.). Novas Metrópoles Paulistas - População, vulnerabilidade e segregação. 1. ed. Campinas: Núcleo de Estudos de População 1(2006).

Recebido em outubro de 2015.

Aceito em abril de 2016. 